\title{
Erratum
}

\section{Horizontal interactions and their symmetries in GUTs}

D.K. Grech

Z. Phys. C - Particles and Fields 42 (1989) 599

The following errors were noticed after the article had been published:

Equation (4.4) should read

$\frac{M_{H}}{M_{x}}=\left(\frac{M_{w}}{M_{x}}\right)^{\beta_{3}^{\mathrm{I}} /\left(\beta_{3}^{\mathrm{I}}-\beta_{3}^{\mathrm{II}}\right)} \exp \left\{\frac{2 \pi\left(\alpha_{3}^{-1}\left(M_{x}\right)-\alpha_{3}^{-1}\left(M_{w}\right)\right)}{\beta_{3}^{\mathrm{I}}-\beta_{3}^{\mathrm{II}}}\right\}$.

Equation (4.7) should read

$\frac{1}{(4 \pi)^{3}}\left|\beta_{333}^{\mathrm{II}}\right| \alpha_{3}^{2}\left(q^{2}\right) \leqq 10^{-n} \frac{1}{(4 \pi)^{2}}\left|\beta_{33}^{\mathrm{II}}\right| \alpha_{3}\left(q^{2}\right)$.
Equation (5.5) should read

$\frac{\hat{M}_{x}^{\prime}}{\widehat{M}_{x}}=\left(\frac{\hat{M}_{x}}{\hat{M}_{H}}\right)^{\left.\left(\beta_{3}^{\mathrm{II}}-3 / 8 \beta_{2}^{\mathrm{II}}-5 / 8 \beta_{1}^{\mathrm{II}}\right) / \tilde{\beta}_{3}^{\mathrm{II}}-3 / 8 \tilde{\beta}_{2}^{\mathrm{II}}-5 / 8 \tilde{\beta}_{1}^{\mathrm{II}}\right)-1}$.

On p. 611 , the second line after formula (4.21) should read:

$S U$ (5) model with $g_{N}$ generations.

On p. 612 the line after formula (5.6) should read:

where $\alpha_{0}^{-1}\left(M_{w}\right)=127.82 \ldots$ 